\title{
PREVALENCIA DA INFECÇÃO POR TRYPANOSOMA CRUZI, EM 1975, EM DOIS BANCOS DE SANGUE DE LONDRINA, PARANÁ, BRASIL
}

José Luís da Silveira Baldy* Lauro Takaoka*

José Dirceu Pereira**

Arnaldo Antônio Calixto ***

Eliane de Fátima Duarte $* * * *$

Baldy, J. L. da S. et al. Prevalência da infecção por Trypanosoma cruzi, em 1975, em dois bancos de sangue de Londrina, Paraná, Brasil. Rev. Saúde públ., S. Paulo, 12:409-16, 1978.

RESUmo: Foi realizado inquérito sobre a positividade de reaçóes sorológicas para o diagnóstico de tripanossomiase americana, em 4.500 candidatos a doadores não selecionados, atendidos em 1975, em dois bancos de sangue de Londrina, Paraná, Brasil. Observoul-se resultado positivo da reação de fixação do complemento em $299(7,9 \%)$ dos 3.774 candidatos a doadores atendidos no Banco de Sangue do Hospital Universitário (individuos residentes predominantemente na zona rural), tendo também sido positivos os resultados da reação de fixação do complemento $e$ do teste de imunofluorescência indireta em 38 (5,2\%) dos 726 candidatos a doadores atendidos no Instituto de Hematologia e Hemoterapia (individuos residentes predominantemente na zona urbana). Na casuística global a positividade observada foi de $7,4 \%$. Dentre os 337 candidatos a doadores com reação sorológica positiva, $97(28,7 \%)$ informaram ter doado sangue anteriormente, em outro local, $71(21,0 \%)$ dos quais em periodo prévio menor que um ano, e $42(12,4 \%)$ em periodo prévio menor que seis meses, em relação $\grave{a}$ data do nosso exame. Comparando os dados obtidos nesta avaliacão com os de inquéritos semelhantes efetuados em $1958 \mathrm{em}$ bancos de sangue deste municipio, concluiu-se que não houve, nesse periodo, alteração significativa nos indices de infeçāo por Trypanosoma cruzi em Londrina. Chamam a atenção para a discrepância entre o reduzido número de casos de doença de Chagas pós-transfusional relatados na literatura $e$ os altos indices de positividade de reações sorológicas para o diagnóstico de tripanossomiase americana registrados em bancos de sangate de diversas regióes do Brasil. Foi ressaltada, a importância de exigir-se maior rigor e parcimônia nas indicaçôes de transfusôes de sangue, e dada ênfase às normas que devem ser respeitadas quando o uso desse recurso terapêtutico tiver indicação formal.

Unitermos: Tripanossomiase americana. Transfusão de sangue. Bancos de sangue, Londrina, PR, Brasil. Inquéritos sorológicos.

- Do Departamento de Clínica Médica. Centro de Ciências da Saúde (CCS). Universidade Estadual de Londrina (UEL). Caixa Postal 2111 - 86.100 - Londrina, PR - Brasil.

* Do Departamento de Patologia Clínica do CCS/UEL

* * Do Banco de Sangue do Hospital Universitário de Londrina do CCS/UEL e do Instituto de Hematologia e Hemoterapia de Londrina.

**** Sextanista do Curso de Medicina da UEL. 
BALDY, J. L. da S. et al. Prevalência da infeç̧ão por Trypanosoma cruzi, em 1975, em dois bancos de sangue de Londrina, Paraná, Brasil, Rev. Saúde públ., S. Paulo, 12:409-16, 1978.

\section{NTRODU Ç A O}

A possibilidade de a doença de Chagas ser transmitida por transfusão de sangue foi, no Brasil, originaimente sugerida por Dias ${ }^{19}$, em 1945. Desde 1952, com a publicação dos primeiros casos humanos da infecção pós-transfusional por Trypanosoma cruzi $^{22}$, a importância desse mecanismo transmissor acha-se cabalmente demonstrada, e invoca-se a prevalência de reações sorológicas positivas em candidatos a doadores de bancos de sangue como um dos indices de risco de transmissão ${ }^{43}$.

Inquéritos realizados, a partir de 1949 , em bancos de sangue de cidades localizadas em diversas regiões do Brasil, evidenciaram prevalências que variaram desde $0,0 \%$ em Santos-SP, até $21,1 \%$ em Ribeirão Preto-SP 11 (Tabela 1).

Se bem que tenham passado 25 anos, desde quando Freitas e col.22 fizeram os primeiros registros dessa ocorrência, não chega a 50 o número de casos de doença de Chagas por transfusão de sangue relatados na literatura (Tabela 2). Como enfatizaram Amato Neto e Dias 4 e Cerisola e col. ${ }^{16}$, esse número, por certo, está muito longe de corresponder à realidade.

Escassas são as publicaçōes sobre a observação da tripanossomíase americana, na fase aguda, no Estado do Paraná. Os primeiros relatos, com comprovação parasitológica, foram os de Amato Neto ${ }^{3}$, em 1958; novos sete casos, autóctones do Paraná, cuja transmissão também se dera pelo vetor habitual, foram descritos por Baranski e col.s, em 1965. Só recentemente, em 1976 , foram relatados os primeiros casos agudos de doença de Chagas por transfusão de sangue, ocorridos no Estado do Paraná i. Por outro lado, Queiroz e Pascual 40 e Brofman 12, em 1958, em inquéritos realizados em dois bancos de sangue de Londrina-PR, evidenciaram positividade sorológica em, respectivamente, $6,9 \%$ e $7,0 \%$ dos candidatos a doadores examinados.
Considerando-se que a doença de Chagas é uma das mais importantes endemias brasileiras ${ }^{39}$, havendo em nosso pais aproximadamente quatro milhões de indivíduos infectados ${ }^{31}$; que são poucas as referências a respeito da situação atual da tripanossomíase americana no Estado do Paraná; que é muito recente, de 1976 , o relato dos primeiros casos de doença de Chagas aguda pós-transfusional neste Estado ${ }^{7}$; e em face de o Norte do Paraná ter sofrido grande mudança em sua ecologia, sobretudo como consequiência da intensa migração interna, derrubadas de matas e diversificação da agricultura, consideramos oportuno publicar os dados deste inquérito.

\section{MATERIAL E METODOS}

Foi feita avaliação dos resultados das reações sorológicas para o diagnóstico de tripanossomiase americana, realizadas em 4.500 candidatos a doadores atendidos em 1975 no Banco de Sangue do Hospital Universitário de Londrina e no Instituto de Hematologia e Hemoterapia de Londrina, Paraná. Nos 3.774 candidatos a doadores atendidos no Hospital Universitário foi feita reação de fixação do complemento e nos 726 candidatos do Instituto de Hematologia foram realizados testes de imunofluorescência indireta e reação de fixação do complemento. Os candidatos a doadores do Banco de Sangue do Hospital Universitário eram familiares de doentes internados ou pessoas de seu relacionamento, oriundos em maioria da zona rural do Norte do Paraná, enquanto no Instituto de Hematologia os candidatos a doadores eram individuos gratificados, que acorreram ao local da coleta espontaneamente ou quando convocados publicamente, sendo de forma invariável moradores da zona urbana do municipio de Londrina. 
BALDY, J, L. da S. et al. Prevalência da infecção por Trypanosoma oruzi, em 1975, em dois bancos de sangue de Londrina, Paraná. Brasil. Rev. Saúde públ., S. Paulo, 12:409-16, 1978.

\section{TA B E L A 1}

Positividade de reações sorológicas para tripanossomiase americana em canđidatos a doadores de sangue, em diversas localidades do Brasil.

\begin{tabular}{|c|c|c|c|c|}
\hline Localidade & Autor(es) & Ano & $\begin{array}{l}\text { Número } \\
\text { de casos }\end{array}$ & $\begin{array}{c}\% \text { de } \\
\text { positividade }\end{array}$ \\
\hline Belo Horizonte-MG & Pellegrino 36 & 1949 & 170 & 1.7 \\
\hline $\begin{array}{l}\text { Belo Horizonte-MG } \\
\text { Hospital do Pronto } \\
\text { Socorro }\end{array}$ & Pellegrino 36 & 1951 & 576 & 2,4 \\
\hline $\begin{array}{l}\text { São Paulo-SP } \\
\text { Hospital Municipal }\end{array}$ & Faria 20 & 1951 & 92 & 7.6 \\
\hline $\begin{array}{l}\text { São Paulo-SP } \\
\text { Hospital das Clínicas } \\
\text { Banco de Sangue de } \\
\text { São Paulo }\end{array}$ & Freitas e col.23 & 1952 & 826 & 2,5 \\
\hline $\begin{array}{l}\text { Ribeirão Preto-SP } \\
\text { Hospital Săo Francisco }\end{array}$ & Biancalana e col.11 & 1953 & 20 & 21,1 \\
\hline $\begin{array}{l}\text { Santos-SP } \\
\text { Hospital da Benefi- } \\
\text { cência Portuguesa } \\
\text { Santa Casa }\end{array}$ & $\begin{array}{l}\text { Biancalana e col.11 } \\
\text { Biancalana e col.11 }\end{array}$ & $\begin{array}{l}1953 \\
1953\end{array}$ & $\begin{array}{l}28 \\
67\end{array}$ & $\begin{array}{l}0,0 \\
0,0\end{array}$ \\
\hline $\begin{array}{l}\text { São José do Rio Preto-SP } \\
\text { Santa Casa }\end{array}$ & Biancalana e col.11 & 1953 & 134 & 14,9 \\
\hline $\begin{array}{l}\text { Araguari-MG } \\
\text { Banco de Sangue }\end{array}$ & Biancalana e col.11 & 1953 & 233 & 19,1 \\
\hline $\begin{array}{l}\text { São Paulo-SP } \\
\text { Santa Casa }\end{array}$ & Passalacqua e col.35 & 1953 & 536 & 4,1 \\
\hline $\begin{array}{l}\text { São Paulo-SP } \\
\text { Não especificado }\end{array}$ & Almeida e col.2 & 1954 & 786 & 5,4 \\
\hline $\begin{array}{l}\text { São Paulo-SP } \\
\text { Hospital das Clínicas }\end{array}$ & Nussenzweig e col.3s & 1955 & 178 & 1,7 \\
\hline $\begin{array}{l}\text { Recife-PE } \\
\text { Banco de Sangue do } \\
\text { Recife }\end{array}$ & Silva e Lima 46 & 1956 & 237 & 3,6 \\
\hline $\begin{array}{l}\text { Londrina-PR } \\
\text { Santa Casa } \\
\text { Casa de Saúde } \\
\text { São Leopoldo }\end{array}$ & Brofman 12 & 1958 & 1.330 & 6,9 \\
\hline $\begin{array}{l}\text { Jaçanã-SP } \\
\text { Hospital São Luiz } \\
\text { Gonzaga }\end{array}$ & Castro e Uvo 15 & 1958 & 627 & 2,0 \\
\hline $\begin{array}{l}\text { Belo Horizonte-MG } \\
\text { Oito bancos de sangue }\end{array}$ & Pellegrino $3 i$ & 1959 & 10.982 & 6,7 \\
\hline $\begin{array}{l}\text { Uberaba-MG } \\
\text { Banco de Sangue Central }\end{array}$ & Jatene e Jacomo 26 & 1959 & 640 & 15,0 \\
\hline $\begin{array}{l}\text { Ribeirão Preto-SP } \\
\text { Hospital das Clínicas } \\
\text { Santa Casa } \\
\text { Força Pública }\end{array}$ & $\begin{array}{l}\text { Freitas e Siqueira 21 } \\
\text { Freitas e Siqueira 21 } \\
\text { Freitas e Siqueira 21 }\end{array}$ & $\begin{array}{l}1959 \\
1959 \\
1959\end{array}$ & $\begin{array}{r}3.055 \\
6.405 \\
626\end{array}$ & $\begin{array}{l}14,4 \\
10,8 \\
10,9\end{array}$ \\
\hline $\begin{array}{l}\text { São Paulo-SP } \\
\text { Hospital das Clínicas }\end{array}$ & Mellone e col.30 & 1960 & 16.624 & 1,5 \\
\hline
\end{tabular}

(continua) 
BALDY, J. L. da S. et al. Prevalência da infeção por Trypanosoma cruzi, em 1975, em dois bancos de sangue de Londrina, Paraná, Brasil. Rev. Saúde públ., S. Paulo, 12:409-16, 1978.

T A B E A 1 (cont.)

\begin{tabular}{|c|c|c|c|c|}
\hline Localidade & Autor & Ano & $\begin{array}{l}\text { Número } \\
\text { de casos }\end{array}$ & $\begin{array}{c}\% \text { de } \\
\text { positividade }\end{array}$ \\
\hline $\begin{array}{l}\text { Rio de Janeiro-RJ } \\
\text { Hospital Såo Francisco } \\
\text { de Assis }\end{array}$ & Silva e col.45 & 1961 & 435 & 1.8 \\
\hline $\begin{array}{l}\text { São Paulo-SP } \\
\text { Hospital das Clínicas }\end{array}$ & Mellone e Pagenotto :" & 1965 & 62.575 & 1.4 \\
\hline $\begin{array}{l}\text { Goiânia-GO } \\
\text { Instituto de Hemoterapia }\end{array}$ & Alexandre I & 1965 & 1.474 & 11.0 \\
\hline $\begin{array}{l}\text { Rio de Janeiro-RJ } \\
\text { Dois bancos de sangue } \\
\text { Banco de Sangue Santa } \\
\text { Catarina }\end{array}$ & $\begin{array}{l}\text { Coura e col.1s } \\
\text { Gonzaga e col.24 }\end{array}$ & $\begin{array}{l}1966 \\
1967\end{array}$ & $\begin{array}{r}4.595 \\
25.508\end{array}$ & $\begin{array}{l}1.2 \\
0.5\end{array}$ \\
\hline $\begin{array}{l}\text { Fortaleza-CE } \\
\text { Faculdade de Medicina } \\
\text { Maternidade }\end{array}$ & $\begin{array}{l}\text { Lima e col.2 } \\
\text { Lima e e el. }{ }^{-}\end{array}$ & $\begin{array}{l}1967 \\
1967\end{array}$ & $\begin{array}{l}267 \\
420\end{array}$ & $\begin{array}{l}5,2 \\
8,6\end{array}$ \\
\hline $\begin{array}{l}\text { Recife-PE } \\
\text { Hospital das Clinicas }\end{array}$ & Huggins e col.25 & 1970 & 136 & 4,4 \\
\hline $\begin{array}{l}\text { Belo Horizonte-MG } \\
\text { Hospital das Clínicas }\end{array}$ & Tavares $+\bar{i}$ & 1971 & 45.236 & 2.5 \\
\hline $\begin{array}{l}\text { São Paulo-SP } \\
\text { Hospital das Clínicas }\end{array}$ & Meira e col.2s & 1972 & 15.341 & 1.9 \\
\hline $\begin{array}{l}\text { Ribeirăo- Preto-SP } \\
\text { Hospital das Clínicas }\end{array}$ & Volpon e col.48 & 1972 & $\begin{array}{l}3.493 \\
4.147\end{array}$ & $\begin{array}{ll}9,5 & (1960) \\
4,3 & (1970)\end{array}$ \\
\hline $\begin{array}{l}\text { Goiania-GO } \\
\text { Hospital das Clínicas }\end{array}$ & Campos e col.1t & 1975 & 4.372 & 10,4 \\
\hline
\end{tabular}

TABELA 2

Casos relatados de doença de Chagas por transfusão de sangue.

\begin{tabular}{|c|c|c|c|}
\hline Ano & Autor(es) & Local & $\begin{array}{l}\text { Número } \\
\text { de casos }\end{array}$ \\
\hline 1952 & Freitas e col.22 & São Paulo-SP & 2 \\
\hline 1955 & Nussenzweig e col.33 & São Paulo-SP & 1 \\
\hline 1955 & Peñalver (citado por Pessôa e Martins 39) & Panamá & 1 \\
\hline 1958 & Votzuk e col. (citado por Cerisola e col.16) & Argentina & 2 \\
\hline 1958 . & Amato Neto 3 & São Paulo-SP & 3 \\
\hline 1962 & Salazar e col.4t & Venezuela & 3 \\
\hline 1963 & Amato Neto e col.5 & Såo Paulo-SP & 1 \\
\hline 1966 & Coura e col.18 & Rio de Janeiro-RJ & 3 \\
\hline 1968 & Amato Neto e col.6 & São Paulo-SP & 3 \\
\hline 1969 & Amato Neto e Dias 4 & São Paulo-SP & 1 \\
\hline 1972 & Bergoglio 10 & Argentina & 18 \\
\hline 1974 & Camargo e Leser 13 & São Paulo-SP & 2 \\
\hline 1975 & Becker 9 & Ribeirão Preto-SP & 1 \\
\hline 1976 & Baldy e col. 7 & Londrina-PR & 3 \\
\hline Total & & & 44 \\
\hline
\end{tabular}


BALDY, J. L. da $\mathrm{S}$, et al. Prevalência da infeç̧ão por Trypanosoma cruzi, em 1975, em dois bancos de sangue de Londrina, Paraná, Brasil. Rev. Saúde públ., S. Paulo, 12:409-16. 1978.

\section{RESULTADOS}

A reação de fixação do complemento para doença de Chagas, realizada no soro de 3.774 candidatos a doadores do Banco de Sangue do Hospital Universitário de Londrina foi positiva em 299 (7,9\%). Setenta e cinco $(25,0 \%)$ desses indivíduos referiram ter doado sangue previamente em outro local; dentre eles, $30(10,0 \%)$ o haviam feito no período de seis meses, e 53 $(17,7 \%)$ no período de um ano antes da realização do nosso exame.

No Instituto de Hematologia e Hemoterapia de Londrina, registrou-se positividade da reação de fixação do complemento e do teste de imunofluorescência indireta em $38(5,2 \%)$ dos 726 candidatos a doadores atendidos. Vinte e dois $(57,8 \%)$ referiram ter doado sangue anteriormente em outro local; dentre eles, $12(31,5 \%)$ o haviam feito no período de seis meses, e $18(47,3 \%)$ no período de um ano antes da realização do nosso exame.

Em relação aos 4.500 candidatos a doadores da casuística global, o indice de prevalència da infecção por Trypanosoma cruzi foi de $7,4 \%$. Noventa e sete $(28,7 \%)$ dos 337 individuos com reação sorológica positiva informaram ter doado sangue anteriormente, e, dentre eles, $71 \quad(21,0 \%)$ tinham feito doação de sangue no período de um ano, e $42(12,4 \%)$ no periodo de seis meses que antecedeu a realização do nosso exame.

\section{COMENTARIOS E CONCLUSOEES}

O local de residència dos candidatos a doadores (predominantemente rural, no Hospital Universitário, e predominantemente urbano, no Instituto de Hematologia) permite-nos considerar que a prevalência de $7,4 \%$ da positividade de reações sorológicas para o diagnóstico da tripanossomíase americana, registrada em nosso inquérito, constitui um índice preciso da situação epidemiológica atual dessa infecção no município de Londrina-PR.
Nossos dados, obtidos em 1975, juntamente com os de Brofman ${ }^{12}$ e de Queiroz e Pascual 40, de 1958, indicam que a prevalência da infecção chagásica - avaliada por intermédio de reações sorológicas em bancos de sangue - não sofreu modificação significativa nesse período, em Londrina-PR. Considerando-se esse fato e os dados da Tabela 1, e conhecendo-se a possibilidade de eventuais resultados negativos da reação de fixação do complemento em indivíduos comprovadamente infectados 14,33,41,42, temos de admitir que o relato de apenas três casos da doença de Chagas aguda pós-transfusional no Estado do Paraná ?, entre os 44 casos registrados na literatura (Tabela 2), não deve corresponder ao que tem ocorrido na realidade. Serve também de apoio a essa argumentação a frequiência muito alta de doação de sangue anterior $(28,7 \%)$ entre os doadores infectados de nossa casuística, que alcançou $57,8 \%$ nos indivíduos com sorologia positiva atendidos no Instituto de Hematologia (doação gratificada). Com base nesses dados, é justo presumir que deve ser elevado o número de candidatos a doadores - especialmente os remunerados, que procuram os bancos de sangue particulares - que omitem 0 resultado de testes sorológicos feitos em outras oportunidades. Levando-se também em conta que as infecções agudas por Trypanosoma cruzi - assintomáticas ou oligossintomáticas na maioria dos pacientes - são clinicamente diagnosticáveis em pequena percentagem de casos (em somente $5 \%$ dos infectados, segundo Cerisola ${ }^{16}$ e col.) parece-nos bem fundamentada a hipótese de que a ocorrência da infecção chagásica por transfusão de sangue deve ser muito mais comum em nosso país.

Sendo, pois, diversos os fatores que aumentam o risco de transmissão da tripanossomíase americana por intermédio de transfusão de sangue - nem todos controláveis com rigor absoluto - , impõe-se submeter as indicações de transfusão de sangue a rigorosa crítica. $O$ uso terapêutico de sangue e de seus derivados deve 
BALDY, J. L. da S. et al. Prevalência da infeçäo por Trypanosoma cruzi, em 1975, em dois bancos de sangue de Londrina. Paraná, Brasil. Rer. Saúde públ., S. Paulo, 12:409-16. 1978.

ser parcimonioso, reservado para os casos em que há indicação formal. Nessas eventualidades, deverá sempre exigir-se a realização prévia, com métodos rigorosamente padronizados, de testes sorológicos para o diagnóstico da tripanossomiase americana; por outro lado, empregar-se-á exclusivamente sangue adicionado com violeta de genciana $16.26 .32-34.42$ nas transfusões efetuadas em áreas endênicas da doença de Chagas, ou em qualquer local onde não se possa dispor de recursos para avaliação sorológica de todos os candidatos a doadores. Mais uma vez, é oportuno lembrar a possibilidade de eventuais resultados negativos, mesmo quando se utilizam técnicas adequadas, en testes de fixação do complemento feitos com sangue de doadores comprovadamente infectados 14.33.11.42. Nas transfusōes de urgência, particularmente de sangue fresco, em que não há tempo para realização de testes sorológicos dos novos doadores, deve-se recorrer a doadores cadastrados, com reações sorológicas negativas no último controle.

Sempre que indicar transfusão de sangue - sobretudo quando houver desrespeito a essas normas -, o médico brasileiro, especialmente o que trabalha nas áreas endèmicas de doença de Chagas, deve estar atento à possibilidade da ocorrência de infecções agudas pós-transfusionais por Trypanosoma cruzi com vista à realização de diagnóstico rápido e preciso, e imediata indicação de terapêtutica especifica ${ }^{3}$.

Bal.DI, J. L. da S. et al. IPrevalence of infection by Trypanosoma cruzi in 1975, in two blood banks in Londrina, Parand, Brazil] Rev. Saúde públ., S. Paulo, $12: 409-16,1978$.

ABSTRACT: The results of an American Trypanosomiasis serologic survey, carried out in 1975 in two blood banks (4,500 unselected blood donor candidates) in Londrina, Paraná (state), Brazil are presented. Positive results were obtained in $299(7.9 \%)$ of 3,774 blood donor candidates observed in the University Hospital and in $38(5.2 \%)$ of 726 candidates observed in Londrina's Hematology and Hemotherapy Institute. Among the 337 positive blood donor candidates, 97 $(28.7 \%)$ were found to have donated blood before, $42(12.4 \%)$ less than six months earlier. There has been no significant change in the positivity of Chagas' disease serological reactions during the last 17 years in Londrina. Attention is called to the discrepancy between the small number of acute cases of Chagas' disease reported and the high rate of positive serologic reactions of American Trypanosomiasis in numerous blood banks in Brazil. Finally, the need to more sparingly prescribe the use of blood transfusions in this country, mainly in the endemic areas of Chagas disease, is emphasized.

Uniterms: Trypanosomiasis, South American. Blood transfusion. Blood banks, Londrina, PR, Brazil. Serological surveys.

\section{REFERENCIAS BIBLIOGRAFICAS}

1. ALEXANDRE, A. apud REZENDE, J. M. et al.42.

2. ALMEIDA. J. O. et al. Complement fixation test with a triple antigen for syphilis. tuberculosis, leprosy or
Chagas disease in blood banks Amer. J. trop. Med. Hyg., 3:490-4, 1954.

3. AMATO NETO, V. Contribuição ao conhecimento da forma aguda da doenca de Chagas. São Paulo, 1958. [Tese Faculdade de Medicina da USP] 
BALDY, J. L. da S. et al. Prevalência da infeç̧ão por Trypanosoma cruzi, em 1975, em dois bancos de sangue de Londrina, Paraná, Brasil. Rev. Saúde públ., S, Paulo, 12:409-16. 1978.

4. AMATO NETO, V. \& DIAS, A. F. Comentảrios sobre caso de transmissão da doença de Chagas por transfusão de sangue e longo periođo de incubação. Rev. Soc. bras. Med. trop., 3:273-5, 1969.

5. AMATO NETO, V. et al. Comprovação de mais um caso de transmissão de doença de Chagas por transfusão de sangue. Hospital, Rio de Janeiro, 64: 123-30, 1963.

6. amato NETO, V. et al. Relato de novos casos de transmissão da doença de Chagas por transfusão de sangue. Rev. Inst. Med. trop. S. Paulo, 10:46-51, 1968.

7. BALDY, J. L. da $S$, et al, Doença de Chagas pós-transfusional: apresentaçăo de três casos. [Apresentado ao 124 Congresso da Sociedade Brasileira de Medicina Tropical, Belém, 1976.]

8. BARANSKI, M. C. et al. Forma aguda de doença de Chagas no Estado do Paraná. Considerações clínicas e epidemiológicas sobre sete doentes. $A n$. Fac, Med. Univ. Paraná, 8:7-24, 1965.

9. BECKER, P. F. L. Moléstia de Chagas aguda acidental por transfusão de sangue de doador chagásico. Rev. Inst. Med. trop. S. Paulo, 17:187-98, 1975.

10. BERGoGLio, R. M. Perfil clínico de la enfermedad de Chagas postransfusional. Rev. méd. Córdoba, 60:123-38, 1972.

11. BIANCALANA, A. et al. Investigações sorológicas sobre doença de Chagas entre candidatos a doadores em bancos de sangue nos Estados de São Paulo e Minas Gerais. Hospital, Rio de Janeiro, 44:745-9, 1953.

12. BROFMAN, $\mathbf{S}$. Incidência da doença de Chagas no Norte do Paraná. Arq. bras. Cardiol., 11:209-10, 1958.

13. CAMARGO, M. E. \& LESER, P. G. Diagnóstico acidental de laboratório de infecções chagásicas agudas pós-transfusionais não suspeitadas. Rev. Ass. méd. bras., 20:335-6, 1974.

14. CAMPOS, C. et al. Prevalência da doença de Chagas no banco de sangue do Hospital das Clinicas de Groiânia. Possibilidade de falha da reação de Guerreiro-Machado na seleção de doađores. Rev. Soc. bras. Med. trop. $9: 165-74,1975$.
15. CASTRO, J. M. \& UVO, D. Incidência da moléstia de Chagas em doadores de sangue no Hospital Săo Luiz Gonzaga, Jaçanã, São Paulo, Arq. bras. Cardiol., 11:114-20, 1958.

16. CERISOLA, J. A. et al. Enfermedad de Chagas y la transfusión de sangre. Bol. Ofic. sanit, panamer., $63: 203-21$, 1972.

17. CERISOLA, J, A, et al. Tratamiento de la enfermedad de Chagas. Buenos Aires, Fundación Rizzuto, 1972.

18. COURA, J. R, et al. fndices de transmissão da doença de Chagas por transfusão de sangue de doadores na fase crônica da doença. Hospital, Rio de Janeiro, 69:991-8, 1966.

19. DIAS, E. Um ensaio de profilaxia da molestia de chagas. Rio de Janeiro, Imprensa Nacional, 1945.

20. FARIA, R. Sífilis, maleita, doença de Chagas e transfusão. Folia clin. biol., $17: 113-7,1951$.

21. FREITAS, J. L. P. \& SIQUEIRA, A. F. Prevalência da infecção chagásica entre candidatos a doakores de sangue e entre outros grupos na cidade de Ribeirão Preto. In: Congresso Internacional sobre Doença de Chagas, Rio de Janeiro, 1959. Resumo de trabalhos. Rio de Janeiro, 1959. p. 20.

22. FREITAS, J. L. P. et al. Primeiras verificações de transmissão acidental da moléstia de Chagas ao homem por transfusão de sangue. Rev. paul. Med. $40: 36-40,1952$.

23. FREITAS, J. L. P. et al. Moléstia de Chagas em bancos de sangue na capital de São Paulo. Hospital, Rio de Janeiro, $41: 229-36,1952$.

24. GONZAGA, A. et al. Rotina sorológica para a doença de Chagas em banco de sangue. Apreciação de resultados na Guanabara de 25.508 reações de fixação do complemento. Arq. bras. Med., 54:289-301, 1967.

25. HUGGINS, D. et al. Inquérito sorológico para o diagnóstico da doença de Chagas entre doadores de um banco de sangue do Recife. Rev. Soc. bras. Med. trop., 4:105-12, 1970.

26. JATENE, A. D. \& JACOMO, R. Doença de Chagas e transfusão de sangue. Rev. goiana Med., 5:23-30, 1959. 
BALDY, J. L. da S, et al. Prevalência da infecção por Trypanosoma cruzi, em 1975, em dois bancos de sangue de Londrina, Paraná, Brasil. Rev. Saúde públ., S. Paulo, 12:409-16, 1978.

27. LIMA, I, M. A. et al. Estudo realizado em doadores de sangue, com respeito à reação de fixação do complemento para doença de Chagas, Rev. Fac. Med. Univ. Fed. Ceara, $7: 3-13,1967$.

28. MEIRA, A. R. et al. Frequiência da reação de Machado-Guerreiro positiva entre os candidatos a doadores de sangue no Hospital das Clínicas da Faculdade de Medicina da Universidade de Săo Paulo em 1970. Rev. Med, 56:327-31, 1972.

29. MELLONE, O. \& PAGENOTTO, J. Incidência de sorologia positiva para sifilis e doenca de Chagas em 62.575 doadores de sangue, Rev. Hosp. Clin. Frac. Med, S. Paulo, 20:165-7, 1965.

30. MELLONE, O. et al. O problema da transmissão da doença de Chagas por transfusão de sangue no Hospital das Clínicas de São Paulo, Res. clin.-cient., $29: 101-2,1960$.

31. MINISTERIO DA SAÚDE. Campanha contra Doença de Chagas: relatório de atividades de 1975. Brasília, 1976.

32. NUSSENZWEIG, V. et al, Ação dos corantes tri-fenil-metânicos sobre o Trypanosoma cruzi in vitro. Emprego da violeta de genciana na profilaxia da transmissão da moléstia de Chagas por transfusão de sangue. Hospital, Rio de Janeiro, 54:25-42, 1953.

33. NUSSENZWEIG, $V$, et al. Moléstia de Chagas em bancos de sangue. Rev. Hosp. Clín. Frac. Med. S. Paulo, 10: 265-83, 1955.

34. NUSSENZWEIG, $V$, et al. Novos dados sobre o emprego de violeta de genciana na profilaxia da transmissão da doença de Chagas por transfusão de sangue. Hospital, Rio de Janeiro, 55: 37-42, 1959.

35. PASSALACQUA, C. S. P. et al. Incidência da doença de Chagas entre candidatos a doadores de um banco de sangue de São Paulo: inquérito sorológico. Hospital, Rio de Janeiro, 43:443-7, 1953.

36. PELLEGRINO, J. Transmissão da doença de Chagas pela transfusão de sangue: primeiras comprovações sorológicas de esquizotripanose em doadores e em candidatos a doadores de sangue. Rer. bras, Med., 6:297-301, 1949.
37. PELlegrino, J. Doença de Chagas e transfusão de sangue. Rev. bras. Malar., 2:697-706, 1959.

38. PELLEGRINO, J. et al. Inquérito sobre a doença de Chagas em candidatos a doadores de sangue. Mem. Inst. Oswaldo Cruz, 49:555-64, 1951.

39. PESSôA, S. B. \& MARTINS, A. V. Parasitologia médica. 9a. ed. Rio de Janeiro, Guanabara Koogan, 1974.

40. QUEIROZ, J. A. \& PASCUAL, J. Contribuição ao estudo da doença de Chagas no Norte do Paraná. Rev. méd. Paraná, $27: 27-30,1958$.

41. RASSI, A. et al. Comportamento evolutivo da reação de fixação do complemento na fase crônica da moléstia de Chagas. Rev. Inst, Med. trop. S. Paulo, $11: 430-5,1969$.

42. REZENDE, $J$. $M$, et al. O problema da transmissão da doença de Chagas por transfusão de sangue. Emprego da violeta de genciana como medida profilática. Rev, goiana Med., 11:35-47, 1965.

43. ROHWEDDER, R. W. Infección chagásica en doadores de sangre y probabilidades de transmitirla por medio de la transfusion. Bol. chil. Parasitol., 24:88-93, 1969.

44. SAlazAR, H. J. et al. Comprobación en Venezuela de la transmisión del Schizotrypanum oruzi por transfusión de sangre. Arch, venez. Med. trop., 4: 355-63, 1962.

45. SILVA, J. R, et al. Investigaçoses sobre a doença de Chagas no Estado da Guanabara: inquérito sorologico entre doadores de sangue e pacientes de ambulatórios. Arq. bras. Med., 51: 35-8, 1961.

46. SILVA, L. H. P. \& LIMA, D. F. Pesquisa da infecção pelo Trypanosoma cruzi entre candidatos a doadores em banco de sangue de Recife-Pernambuco. Publ. méd,, S. Paulo, 27:23-5, 1956.

47. TAVARES, J. A. Reação de Guerreiro e Machado em doadores de sangue. Rev. Ass. méd. Minas Gerais, 22:183-90, 1971.

48. VOLPON, J. B. et al. Moléstia de Chagas no Banco de sangue do Hospital das Clínicas de Ribeirão Preto nos anos de 1960 e 1970. Med. CARL, Ribeirão Preto, 5:77-84, 1972.

Recebido para publicação em 04/11/197\% Aprovado para publicação em 13/04/1978 